\title{
Wild strawberry polyphenols exhibit gut-protective bioactivity following in vivo digestion
}

\author{
Chris Gill ${ }^{1}$, Cheryl Latimer ${ }^{1}$, Nigel Ternan ${ }^{1}$, Kirsty Pourshahidi ${ }^{1}$, Massimilano Fontana ${ }^{1}$, \\ Roger Lawther ${ }^{2}$, Gloria O’Connor ${ }^{2}$, Lorenzo Conterno ${ }^{3,4}$, Francesca Fava ${ }^{3}$ and Kieran Tuohy ${ }^{3}$ \\ ${ }^{1}$ Ulster Univerity, Coleraine, United Kingdom, \\ ${ }^{2}$ Altnagelvin Hospital, Londonderry, United Kingdom, \\ ${ }^{3}$ Fondazione Edmund Mach, San Michele all' Adige, Italy and \\ ${ }^{4}$ Laimburg Research Centre, Laimburg, Italy
}

\section{Abstract}

Berries are one of the most commonly consumed sources of bioactive polyphenols and these compounds may exert protective effects against initiation of colorectal cancer (CRC) by reducing DNA damage. The inverse correlation between fruit and vegetable consumption and the incidence of CRC is well established, hence the role of berry derived bioactive phytochemicals in promotion of gut health is of interest. Ileostomy studies provide a unique insight into food digestion, allowing identification of physiologically relevant dietary phytochemicals and their metabolites. Here, we hypothesised that physiologically relevant levels of Italian wild strawberry metabolites exiting the ileum would be both bioavailable and would in turn exert positive effects on gut health markers.

Five ileostomists completed a wild strawberry feeding study (11/NI/0112), ileal fluid was collected pre $(0 \mathrm{~h})$ and post $(8 \mathrm{~h})$ consumption of strawberries $(225 \mathrm{~g})$ and assessed for phytochemical composition by LCMSn. We simulated the interaction of the ileal fluids with colonic microbiota over a $24 \mathrm{~h}$ period $(0,5,10,24 \mathrm{hr})$ using in vitro gut fermenter models. Nutri-kinetic analysis using LCMSn demonstrated significant increases in the concentration of gut microbiota-mediated polyphenolic metabolites over time, including 3-(4hydroxyphenyl) propionic acid, 3-(3-hydroxyphenyl) propanoic acid, hydroxybenzoic acid and urolithin A. While changes in the bacterial composition of the gut fermenter model(s) were monitored using fluorescent in situ hybridisation analysis (FISH) with validated probes for Total bacteria, Bifidobacterium genus, Clostridium histolyticum/perfringens group, Faecalibacterium prausnitzii, Eubacterium rectale group, Bacteroides, Lactobacilli and Enterobacteria; limited changes were observed.

Bioactivity of the post-berry consumption ileal fermentates was assessed on two colonocyte cell lines (HT29 and CCD841 CON (normal)) using the oxidative challenge COMET assay. Post-berry ileal fermentate $(24 \mathrm{~h})$ from all five ileostomists significantly (p $<0.01)$ decreased DNA damage (expressed as \%Tail DNA) in both HT29 cells $(\sim 45 \%)$ and CCD841 cells ( 25\%) compared to untreated controls.

To conclude, strawberry phytochemicals were available for colonic fermentation following ileal digestion and human microbiotamediated fermentation which subsequently increased overall levels of polyphenolic metabolites, the post berry fermentates were demonstrated to reduce DNA damage in colonocytes.

\section{Conflict of Interest}

There is no conflict of interest. 